\title{
Cuentas satélite de Cultura, apuesta y propuesta iberoamericana para las estadísticas culturales en el mundo
}

\author{
Por Liliana Patricia Ortiz Ospino
}

\section{Resumen}

El presente documento se realizó con el objetivo de conocer las experiencias de las cuentas satélite de cultura (CSC) en Iberoamérica. Para el efecto se diseñaron tres matrices que compilaron información sobre aspectos metodológicos, resultados obtenidos y fuentes de información utilizadas para la construcción de las cuentas en la región. Los resultados de la información evidencian que de los 21 países de la región, 13 han implementado las CSC y tienen resultados consolidados, 5 se encuentran en proceso de implementación y de los restantes no se pudo establecer si están interesados en este tipo de mediciones para el sector cultural. Adicionalmente, se confirmó que las CSC como sistema requiere de ajustes que obligan a tomar acciones para garantizar su sostenibilidad. Uno de los desafíos que enfrenta es el relacionado con las características de la información para lo cual se recomienda un proceso de planificación estadística; otro reto importante es referido a la viabilidad de las comparaciones internacionales del sector que se requieren para avanzar aunando esfuerzo entre países, para lo que se propone la unificación de criterios mediante el desarrollo de un marco conceptual y metodológico de carácter global; y el último, tiene que ver con la utilización de los datos generados en las CSC, para ello se propone emprender una estrategia de divulgación y difusión efectiva de los resultados. No obstante se requieren de estas mejoras, las cuentas satélite de cultura conforman un sistema de información coherente y ordenado que genera datos relevantes de información, y para lberoamérica la apuesta y propuesta de producción para los avances en producción de información cultural para el mundo.

\section{Abstract}

This document was made with objective of knowing the experiences of cultural satellite accounts (CSC, Spanish Acronyms) in Latin America. For this purpose, three matrices were designed to compile information on methodological aspects, results obtained and sources of information used for construction of CSC. Results show the progress of 21 countries from the Region; 13 countries implemented this accounts and have consolidated results, 5 are in process to being implemented and 3 of them, it was impossible to established if they are interested in this type of measurement for Cultural Sector. In addition, it was confirmed that CSC as a system, requires adjustments which force actions for ensure its sustainability. One of this challenges found in research, is related to data characteristics; for this reason, a process of statistical planning is recommended; Another important challenge is viability of the international comparisons of the sector, that required to move forward, bringing together efforts between countries, for which it is proposed to unify criteria by developing a global conceptual and methodological framework; And the last one, is referred to use of the data generated in the CSC. In this way, it suggests to undertake an effective dissemination strategy of results. Despite these improvements, the cultural satellite accounts are a coherent and orderly information system that generates relevant cultural data, and for Latin America are commitment and proposal for the progress in the production of global cultural information.

\section{Resumo}

Este documento foi feito com o objetivo de conhecer as experiências de contas satélite de cultura (CSC) na América Latina. Para este efeito, três matrizes informações compiladas sobre questões metodológicas, resultados e fontes de informação utilizadas para a construção das contas da região foram concebidos. Os resultados dos dados mostram que dos 21 países 
da região, 13 implementaram CSC e ter resultados, cinco estão em processo de implementação e os restantes não foi possível determinar se eles estão interessados neste tipo de medida para a consolidação sector cultural. Além disso, foi confirmado que a CSC como um sistema requer ajustes que obrigam a tomar medidas para assegurar a sua sustentabilidade. Um dos desafios está relacionada com as características da informação para a qual é recomendado um processo de planejamento estatístico; Outro desafio importante é baseada na viabilidade de comparações internacionais do setor que são necessários para avançar união de esforços entre os países em que a unificação de critérios propostos pelo desenvolvimento de um quadro conceitual e metodológico de natureza global; e último, tem a ver com o uso dos dados gerados no CSC, para ele pretende levar a cabo uma estratégia de disseminação e divulgação eficaz dos resultados. No entanto, estas melhorias são necessárias, contas cultura satélite compõem uma informação coerente e ordenado relevante gerado dados de informação, e na América Latina a proposta de aposta e de produção para avanços na produção para a informação cultural mundial. 


\section{Introducción}

Los postulados teóricos basados en modelos de crecimiento han influido hace algunas décadas en el enfoque del sector cultural de manera que, se asume que gran parte del mismo contribuye de manera positiva a la economía en su conjunto, y por tanto este campo se constituye en un generador no sólo de bienestar, sino también de riqueza para la economía nacional (Potts \& Cunningham, 2008).

Basados en este tipo de modelo los gobiernos e instituciones toman sus decisiones de políticas públicas culturales y en consecuencia, impulsan la construcción de sistemas integrados de información que apoyen al sector en la obtención de datos veraces, oportunos y permanentes para conocer el contexto y atender de manera adecuada las problemáticas y necesidades que le aquejan.

En este contexto surge la necesidad de las cuentas satélite de cultura, sistema contable, coherente ordenado cuyos métodos generan información económica monetaria y no monetaria, sobre la oferta y la demanda cultural.

Este documento surge con la necesidad de conocer las experiencias de la construcción de las cuentas satélite de cultura en Iberoamérica, por esta razón se realizó una investigación descriptiva y no exhaustiva de los países de la región que las han implementado.

Las conclusiones sobre estas experiencias confirman que existen tres desafíos importantes que enfrentan estos sistemas. El primero es referido a las características de la información básica que es el insumo para elaboración de las cuentas, el segundo está relacionado con la viabilidad de las comparaciones internacionales y el tercero, con la utilización de los datos generados en las CSC.

Para estos tres desafíos el documento propone tres acciones de política: planificación estadística estratégica para el sector, marco conceptual y metodológico de carácter global y una estrategia efectiva de divulgación y difusión.

\section{Aproximación conceptual a las cuentas satélite de cultura}

El término 'cuentas satélite' genera mucha curiosidad cuando se menciona en el ámbito de la estadística, sin embargo es un concepto utilizado desde hace décadas en el Sistema de Cuentas Nacionales (SCN) para nombrar las extensiones del marco central de las cuentas nacionales, que ofrecen cierta flexibilidad y profundización de aspectos específicos de la vida económica, como sectores o áreas de interés, sin sobrecargar ni distorsionar las características de las cuentas nacionales en su conjunto (Naciones Unidas et al., 2008). 
Existen dos tipos de cuentas satélite, una implica un reordenamiento de las clasificaciones centrales y la posibilidad de introducir elementos complementarios, y la otra se basa en el desarrollo de conceptos alternativos a los propuestos en el SCN (Naciones Unidas et al., 2008).

Las cuentas satélite de cultura (CSC) surgieron en el mundo como una herramienta para realizar mediciones económicas del campo cultural identificando y reorganizando la información dispersa en el marco central y agregando elementos para la generación de información relevante para el sector. Sin embargo, la dinámica de su implementación ha requerido el desarrollo de conceptos alternativos a los del SCN, la noción de campo cultural o la definición de indicadores de empleo y del gasto en cultura.

En este contexto, es conveniente analizar el término campo cultural, que se ha comprendido desde la visión de Bourdieu (1966), como "el sistema de relaciones constituido por los agentes sociales que están directamente vinculados con la producción, la circulación, el consumo y la apropiación de las obras artísticas" (CAB, 2015). Este conjunto de procesos ha sido denominado por el Marco de Estadísticas Culturales (MEC) de UNESCO (2009), como ciclo cultural. Tal como se define, el ciclo cultural abarca el conjunto de etapas de creación, producción, difusión, exhibición, recepción, transmisión, consumo, participación y apropiación de las artes y el patrimonio cultural (MEC, 2009).

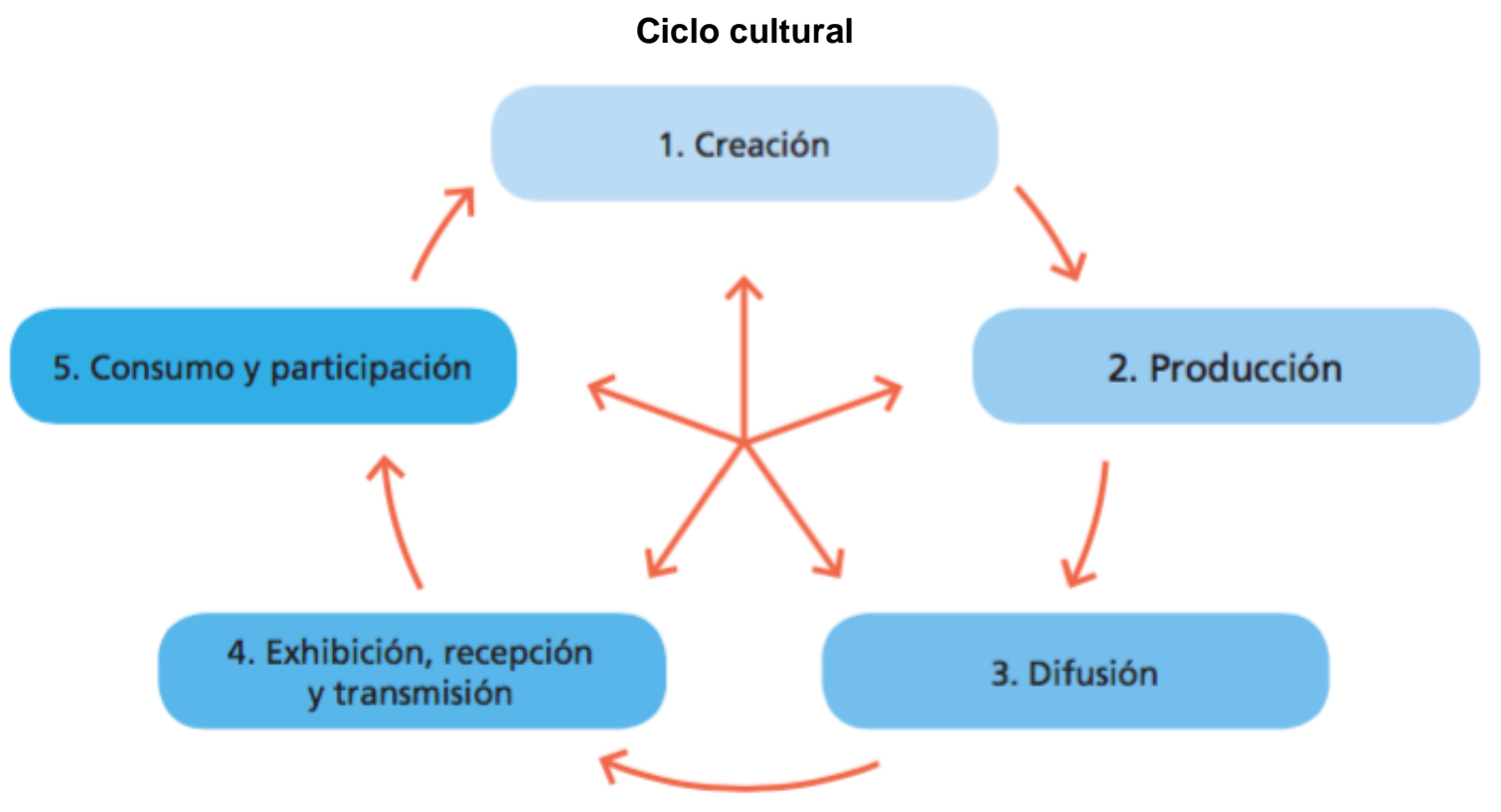

Fuente: Marco de Estadísticas Culturales (UNESCO, 2011)

Este concepto es útil ya que sugiere la existencia de interconexiones entre oferta y demanda, incluyendo procesos de realimentación a través de los cuales el consumo y apropiación cultural inspiran la creación y producción de nuevos productos" (MEC, 2011). Adicionalmente, ayuda a la 
comprensión de la taxonomía del campo cultural y su segmentación para efectos de una medición. De esta manera, el MEC propone una taxonomía con propósitos estadísticos del campo cultural, basada en un marco de dominios, tal como aparece en el siguiente esquema:

\section{Marco de dominios de estadísticas culturales}

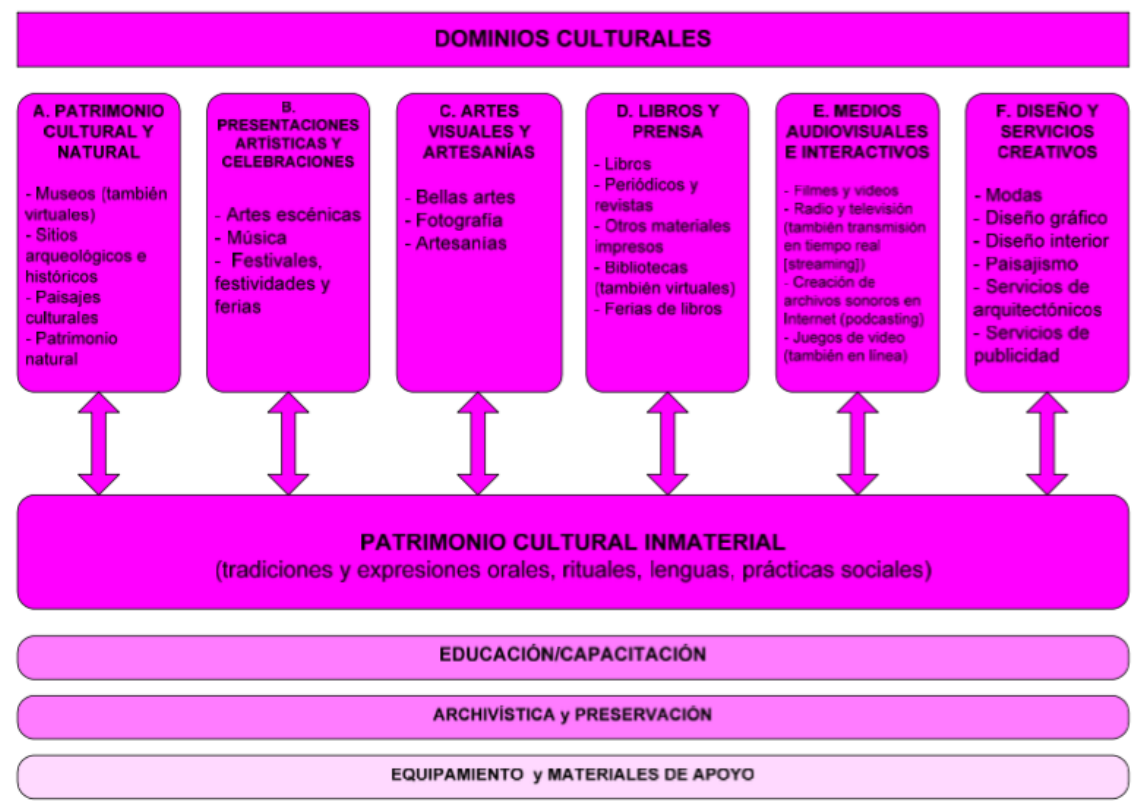

DOMINIOS RELACIONADOS

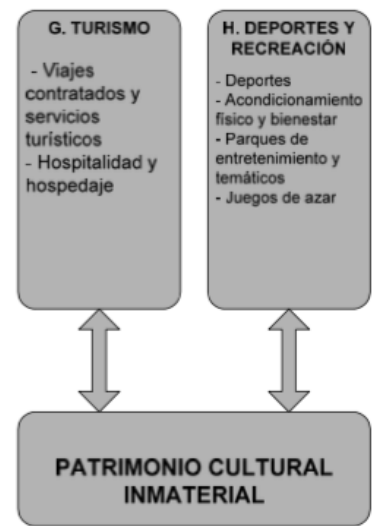

EDUCACIÓN Y CAPACITACIÓN

ARCHIVISTICA Y PRESERVACION

EQUIPAMIENTO Y MATERIALES DE
APOYO

Fuente: UNESCO, 2011

Por otro lado, el Convenio Andrés Bello (2015), sugiere en la Guía Metodológica para la Implementación de las Cuentas Satélite de Cultura en Iberoamérica, una segmentación para la Región con algunas variaciones en sectores y subsectores respecto al MEC, a los que se asocian los productos (bienes o servicios) y las actividades característicos o típicos del campo cultural para las cuentas satélite. Estos sectores y subsectores son:

Tabla de sectores y subsectores del campo cultural

\begin{tabular}{|l|l|}
\hline \multicolumn{1}{|c|}{ Sector } & \multicolumn{1}{c|}{ Subsector } \\
\hline \multirow{4}{*}{$\begin{array}{c}\text { Creación- Derechos de } \\
\text { Autor }\end{array}$} & Literaria \\
\cline { 2 - 2 } & Musical \\
\cline { 2 - 2 } & Audiovisual \\
\hline \multirow{4}{*}{ Diseño } & Arquitectónico \\
\cline { 2 - 2 } & Industrial o de Productos \\
\cline { 2 - 2 } & Gráfico \\
\cline { 2 - 2 } & Textil \\
\cline { 2 - 2 } & de Moda \\
\cline { 2 - 2 } & de Joyas \\
\hline
\end{tabular}




\begin{tabular}{|c|c|}
\hline & Publicitario \\
\hline & Web \\
\hline Juegos y juguetería & Juegos y juguetería \\
\hline & Teatro \\
\hline Artes escénicas y & Danza \\
\hline espectáculos artísticos & $\begin{array}{l}\text { Otras formas de artes escénicas (circo, pantomima, narración, } \\
\text { declamación etc.) }\end{array}$ \\
\hline & Artes plásticas (incluye representaciones de origen mixto) \\
\hline $\begin{array}{c}\text { Artes plásticas y } \\
\text { visuales }\end{array}$ & Fotografía \\
\hline & Artes gráficas e ilustración \\
\hline & Presentaciones musicales en vivo \\
\hline Música & Edición de música \\
\hline & Producción fonográfica \\
\hline & Cine y video (incluye animación) \\
\hline & Radio \\
\hline Audiovisual y Radio & Televisión \\
\hline & Juegos on-line \\
\hline & Videojuegos \\
\hline & Libros \\
\hline Libros y publicaciones & Publicaciones Periódicas \\
\hline & Bibliotecas \\
\hline & Educación cultural no formal (orientada a la lúdica) \\
\hline Educación cultural & $\begin{array}{l}\text { Educación cultural formal (incluye educación superior, formación en } \\
\text { bellas artes, diseño y otros) }\end{array}$ \\
\hline & Entidades museales \\
\hline & Archivos históricos culturales \\
\hline Patrimonio material & $\begin{array}{l}\text { Otro patrimonio inmueble (centros históricos, monumentos históricos, } \\
\text { patrimonio arqueológico) }\end{array}$ \\
\hline & Otro patrimonio mueble (antigüedades, cuadros históricos, etc.) \\
\hline & Fiestas tradicionales y patrias \\
\hline Patrimonin inmaterial & Cocinas tradicionales \\
\hline 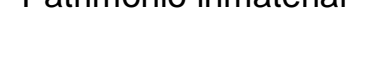 & Artesanías \\
\hline & Lenguas \\
\hline
\end{tabular}

Fuente: CAB, 2015

Con el fin de establecer las asociaciones de productos y actividades característicos a los sectores y subsectores culturales, es necesaria su identificación utilizando las clasificaciones y nomenclaturas internacionales, ejercicio que ayuda a determinar una taxonomía común del sector y además, garantiza la comparabilidad entre países. 
El punto de partida para la construcción de las cuentas satélite de cultura es identificar los productos específicos del área, es decir los característicos o típicos y los conexos, interdependientes y auxiliares (Naciones Unidas et al., 2008). Los productos característicos o típicos son el resultado de los procesos que se dan en el campo cultural. Por ejemplo: libros, espectáculos artísticos, esculturas, exhibición de películas, fotografías, entre otros. A estos bienes o servicios se asocian las actividades características de la cultura.

La Guía del CAB y el MEC proponen la identificación de los productos típicos a partir de la Clasificación Central de Productos (CPC) y de las actividades desde la Clasificación Internacional Industrial Uniforme (CIIU) de Naciones Unidas, preferiblemente en sus últimas versiones o revisiones.

De otra parte, los productos conexos, interdependientes y auxiliares son de interés porque sus usos están claramente comprendidos en el concepto de gasto cultural, es decir aquellos que se requieren para consumir, participar o apropiarse del contenido cultural, pero también los que se necesitan para desarrollar actividades económicas o prácticas culturales (CAB, 2015). Por ejemplo, cámaras fotográficas, proyectores de cine, dispositivos digitales, pinceles, entre otros.

Según la Guía del CAB, el sistema de información de las cuentas satélite comprende:

- Las cuentas de producción y de generación del ingreso

- Los equilibrios oferta-utilización

- El análisis del gasto y su financiación

- El análisis del trabajo y del empleo

- Los indicadores complementarios

Las cuentas de producción y de generación del ingreso de las actividades características, permiten conocer el resultado de los procesos productivos típicos del sector cultural y el valor que agregan estos procesos a la economía en su conjunto. Para esto, relacionan el valor de la producción de un productor o de un grupo de productores, con el valor de los insumos utilizados en el proceso productivo. El saldo obtenido en estas cuentas se llama valor agregado (CAB, 2015).

Los equilibrios oferta-utilización de los productos culturales, representan la oferta y los usos de los bienes y servicios culturales dentro del marco global de una economía, tales como consumo intermedio, consumo final, formación de capital, exportaciones, entre otros; tienen en cuenta no solo aquellos productos culturales producidos o consumidos por residentes, sino también aquellos exportados o importados (CAB, 2015). 
De otra parte, el análisis del gasto presenta las erogaciones en cultura que realizan los agentes residentes (incluyendo la adquisición de bienes y servicios producidos en el exterior) o aquellos que financian este tipo de gasto; en particular, estudia cuál es la importancia del gasto de la autoridad pública o de terceros para facilitar el acceso a la cultura, los mecanismos utilizados y quiénes se benefician directa e indirectamente de estos desembolsos.

El análisis del empleo y del trabajo ayuda a la comprensión de la relación entre las actividades productivas culturales, el empleo y la fuerza de trabajo, y constituye una herramienta para aproximarse a la economía no observada (CAB, 2015). De esta manera, este análisis que se propone en las cuentas satélite de cultura, incorpora datos sobre la economía formal y la informal del sector.

Los indicadores complementarios ofrecen información adicional, monetaria y no monetaria, sobre la oferta y la demanda de bienes y servicios; además permiten contrastar y realimentar la información generada por las cuentas, ya que estas solo se expresan en valores monetarios (CAB, 2015).

En resumen, las cuentas satélite constituyen un sistema contable, coherente y organizado que provee información económica relevante, y apoya la toma de decisiones en el diseño de políticas públicas, en la asignación y distribución del gasto público, y en las estrategias por parte de productores y agentes económicos del sector cultural.

\section{Desarrollos de las CSC en el ámbito mundial}

Los primeros países en aproximarse a los desarrollos de las cuentas satélite de cultura, en los años 70 s y 80 s, fueron Finlandia y Francia; sin embargo, los pioneros en implementarlas y publicar resultados fueron Colombia en 2007 y, Finlandia y España en 2009.

La década actual ha sido prolífica en desarrollos de las CSC, a los países que hicieron sus primeras mediciones se han sumado Estados Unidos, Reino Unido, Australia, República Checa, Polonia, otros de la Región Asia Pacífico (CEPAL \& OEI, 2015) y casi todos los países de la región iberoamericana (CAB, 2015)

Una característica común en estos países es que la mayoría cuentan con alianzas de trabajo entre las instituciones responsables de las políticas culturales, los institutos de estadísticas oficiales y las organizaciones que realizan las cuentas nacionales. Estas asociaciones garantizan intercambio de información, de conocimiento y de experiencia frente a este tipo de mediciones. En Australia, Finlandia y los países de la Región Iberoamericana las alianzas entre el instituto o ministerio de 
cultura y la institución de estadísticas oficiales, permitieron realizar avances significativos en periodos cortos de tiempo.

Existen particularidades en la medición de cada país que han dificultado la comparabilidad de los resultados una de estas se refiere a los marcos conceptuales y metodológicos utilizados. A continuación se hace una breve descripción del estado actual de las experiencias internacionales ajenas a la región iberoamericana:

\section{Oceanía}

Los primeros resultados de las cuentas satélite de cultura del continente australiano fueron publicados en 2014 y corresponden a la serie 2008-2009. Los resultados actuales estiman que la actividad cultural y la creativa contribuyen en promedio con el 6,9\% del Producto Interno Bruto Australiano, mayor que las contribuciones de los sectores de agricultura, transporte, telecomunicaciones y educación. (Australian Bureau of Statistics, 2014).

El campo de medición de las cuentas comprende: museos, patrimonio natural, librerías y archivos, literatura y medios impresos, artes escénicas, diseño, multimedia y películas, composición musical, artes visuales y artesanías, diseño de modas, otros bienes de manufacturas y ventas, y actividades de soporte a la cultura (Australian Bureau of Statistics, 2014); y su alcance temático abarca la medición del producto interno bruto y el empleo (Australian Bureau of Statistics, 2014.

\section{América del Norte}

Canadá y Estados Unidos cuentan con cuentas satélite de cultura con resultados consolidados desde el año 2010 y 2013, respectivamente. En Canadá los sectores estudiados comprenden: patrimonio cultural y librerías, artes visuales y aplicadas, artes escénicas, escritura y publicaciones, educación y entrenamiento, música grabada, audiovisual y multimedia interactiva, y por último gobierno, fundaciones y soporte profesional para la cultura. Su contribución a la economía total fue del 3\% durante el período 2010-2014. Se destaca en estas cuentas la visión nacional y territorial, pues se calcula la contribución en los dos ámbitos (Gobierno de Canadá, consultado el 1 de marzo de 2017).

Por otro lado, en Estados Unidos estas cuentas son llamadas cuentas satélite de las artes y la producción cultural; fueron publicadas en 2014 y cuentan con una serie desde 1998 a 2012 , comprenden los subsectores de: radiodifusión, fotografías e industrias del video, publicaciones, comercio al por menor, artes escénicas y artistas independientes, y publicidad. La contribución al producto interno bruto de Estados Unidos es de 4,3\% (National Endowment for the Arts, 2013).

\section{Europa}


El continente europeo es referente por sus trabajos pioneros de aproximación a las cuentas satélite de cultura, en este grupo se destaca Finlandia con una serie de resultados de los años 1995-2008, ejercicio piloto realizado en 2007, de acuerdo con la Clasificación Industrial Estándar 2002, cuya contribución al Producto Interno Bruto se registró en 3,0\%. Los resultados más recientes (serie 2008-2014) corresponden a la Clasificación 2008 y muestran un aporte a la economía total de 2,9\% (Statistics Finland, 2016).

Las cuentas en este país se elaboraron gracias a la unión de esfuerzos del Ministerio de Educación y Cultura y el Instituto Nacional de Estadísticas de Finlandia y comprenden las actividades artísticas, teatrales y conciertos; Bibliotecas, archivos y museos; Tiendas de arte y antigüedades; actividades de producción y distribución de libros; periódicos; producción y distribución de películas y videos; fabricación y venta de instrumentos musicales; grabaciones musicales; radio y televisión; actividades de impresión; publicidad, diseño arquitectónico e industrial; Fotografía; Parques de diversiones, juegos y otras actividades recreativas; fabricación y venta de artículos electrónicos para entretenimiento; Organización de eventos culturales; Industrias deportivas, educación y administración cultural (Statistics Finland, 2016).

Por su parte, el Reino Unido ha trabajado en las primeras aproximaciones a las cuentas satélite de cultura desde el año 2008 mediante un programa de investigación de los subsectores culturales, que aborda la financiación pública, la contribución económica del sector, el empleo, la producción, el crecimiento, y estimaciones de las contribuciones indirectas como turismo, a cargo del organismo responsable de las políticas culturales en ese país, el Departamento de Cultura, Medios y Deportes (DCMS, por sus siglas en inglés).

Dentro de los dominios de estudios se encuentran las antigüedades, museos y galerías, exportación de objetos de interés cultural, juegos, industrias creativas, telecomunicaciones; como dominios alternativos se encuentran los deportes y el turismo (DCMS, 2016). Las series calculadas son de los años 2008 a 2014 y fueron publicados por el DCMS en octubre de 2016 y reportan una contribución de la cultura al producto interno bruto de Reino Unido estimado en el 13,3\%. Asimismo presentan exportaciones del 17,2\% de las exportaciones totales del país e importaciones del $14,6 \%$ del total de las importaciones nacionales (DCMS, 2016).

La República Checa publicó los primeros resultados de las cuentas satélite en el año 2012, esta comprende los subsectores de patrimonio, artes escénicas, artes visuales, libros y prensa, tecnologías audiovisuales e interactivas, arquitectura, publicidad y educación cultural. Para la ocasión se calcularon las series 2009-2010 abordando el análisis de empleo, gasto público, inversión, importación, exportación, ingresos, consumo intermedio, consumo de los hogares, valor agregado bruto y Producto Interno Bruto Cultural (CEPAL \& OEI, 2015). 
Rumania es otro de los países europeos con desarrollo de cuentas satélite de cultura, la cual se elaboró en coordinación con la Oficina de Derecho de Autor de Rumania, el Centro de Estudios e Investigación en el Campo de la Cultura y el Instituto Nacional de Economía. Estas tres entidades trabajaron durante 2008 en el cálculo del valor agregado de las actividades culturales, del cual se obtuvo una contribución del 3,8\% en la economía total del país (Gobierno de Rumania, consultado el 2 de marzo de 2017).

Los resultados en este país corresponden al período 2000-2005 y los sectores contemplados fueron: producción de videos y películas de cine, creación literaria y artística, artes escénicas, productos de consultoría y software, publicidad, edición de música grabada, actividades de impresión, radio y televisión, edición de programas, publicación de periódicos y de libros. De estos sectores se calculan la producción, el valor agregado y el empleo.

\section{$\underline{\text { Asia }}$}

El grupo de países de la red de Asia Pacífico están trabajando en el desarrollo de mediciones del sector cultural mediante el marco ofrecido por las cuentas satélite, sin embargo aún no se tienen resultados oficiales de estas mediciones.

Los datos de las cuentas en cada continente permiten evidenciar que se tienen diferencias en el campo de medición, las clasificaciones y los marcos conceptuales y metodológicos que se han tomado como base para la elaboración de las cuentas satélite, además los años utilizados también varían de un país a otro. De esta manera, y a pesar de la acogida de las cuentas satélite de cultura en el ámbito internacional, las comparaciones internacionales resultan aún inviables porque no existe un marco metodológico que permita un desarrollo unificado de las cuentas; esto constituye un desafío cuya superación permitiría recomendar acciones y estrategias para el diseño y análisis de políticas públicas culturales de carácter global y regional.

\section{Metodología para la investigación de experiencias de las CSC en Iberoamérica}

Este documento de trabajo surge con la finalidad de conocer la situación actual de implementación de las cuentas satélite de cultura en lberoamérica, los logros obtenidos con este tipo de mediciones y los desafíos que enfrentan los responsables de su elaboración.

Para el efecto, se realizó una investigación en los países de la región mediante la recolección de información pública oficial consultada en documentos, noticias y notas de páginas web, informes de prensa o piezas comunicativas de las entidades responsables de su elaboración o difusión en cada país, es decir de los ministerios o instituciones de la cultura, los institutos de estadísticas oficiales o entidades multilaterales que han apoyado la elaboración de las cuentas satélite en la 
región, tales como Organización de los Estados Iberoamericanos (OEI), Organización de los Estados Americanos (OEA), Convenio Andrés Bello (CAB), entre otros.

Con este propósito se diseñaron 3 matrices que apoyaron la indagación de características de las cuentas satélite, de acuerdo con los avances realizados por los países de la región. La primera matriz reunió datos metodológicos de las cuentas satélite, la segunda información sobre los resultados obtenidos por los países en sus mediciones y la tercera, datos relacionados con las fuentes de información utilizadas para la elaboración de las cuentas y las entidades responsables de su generación.

El análisis de la información fue meramente descriptivo, utilizando las herramientas de metodología cualitativa, por esta razón una porción de él se hizo a través del programa NVivo, que permitió la identificación de palabras más frecuentes y de información clave para la descripción de las cuentas satélite de cada país.

En la recolección de datos se tuvo ciertas limitaciones debido a que en algunos de los documentos no se colocaron las fechas de publicación, o los resultados fueron divulgados mediante ruedas de prensa y boletines informativos que no especifican la metodología utilizada, las clasificaciones o marcos conceptuales. Algunos datos sobre los países no tienen fácil acceso en las instituciones encargadas de su divulgación y difusión, lo que implicó una búsqueda avanzada para su ubicación.

\section{Avances de implementación de las CSC en lberoamérica}

Los esfuerzos de Gobiernos iberoamericanos y de organismos multilaterales que intervienen en la región, se han enfocado en el desarrollo de sistemas de información cuya generación de datos les permita tomar decisiones adecuadas en las políticas públicas del sector. Por esta razón las iniciativas del Banco Interamericano de Desarrollo (BID), Organización de los Estados Americanos (OEA), Organización de los Estados Iberoamericanos (OEI), Convenio Andrés Bello (CAB), Comunidad Andina de Naciones (CAN), Coordinación Educativa y Cultural Centroamericana (CECC/SICA), Sistema de Información Cultural de Mercosur (SICSUR) y la Comisión Económica para América Latina y el Caribe (CEPAL) han recomendado y apoyado este tipo de mediciones.

En este contexto se presentan los datos obtenidos en la investigación realizada sobre las experiencias en lberoamérica, de los 21 países que conforman la región. De esta manera se pudo establecer que existen 13 de ellos que han implementado las cuentas satélite de cultura y cuentan con resultados consolidados, 5 en proceso de implementación o interesados en su elaboración y 3 de los que no fue posible determinar si existe interés en este tipo de mediciones o han avanzado en su elaboración. 


\subsection{Países con implementación y resultados}

Los países que conforman el primer grupo son: Argentina, Bolivia, Chile, Colombia, Costa Rica, Ecuador, España, Guatemala, México, Perú, Portugal, República Dominicana y Uruguay. La mayoría de estos países tienen publicados sus datos y notas metodológicas en medios masivos de divulgación como páginas web, informes de prensa o piezas comunicativas, sin embargo países como Ecuador, Bolivia y Perú, publicaron sus datos en conjunto como cuentas satélite del Área Andina y por esto sus notas metodológicas y resultados aparecen en una sola publicación.

Para este primer bloque de países se recolectó la información de las tres matrices propuestas:

\subsubsection{Matriz de aspectos metodológicos}

Esta matriz comprende las variables: fuente de consulta, entidades responsables de la elaboración de las cuentas, bases metodológicas utilizadas, objetivo, alcance temático, limitaciones de la medición y desafíos.

Con relación a las entidades responsables del desarrollo de las cuentas satélite de cultura en Iberoamérica, la situación no es muy distinta a las experiencias de otros continentes. En la mayoría de los países se encontró que la responsabilidad de su elaboración recae en los ministerios, institutos o secretarías de cultura en alianza con las instituciones de estadísticas oficiales. Países como Chile, Costa Rica, y República Dominicana establecieron además convenios con el Banco Central del país, entidad que en ocasiones es la encargada del desarrollo de cuentas nacionales y cuentas satélite.

Dentro de las bases conceptuales y metodológicas se tienen el MEC y las metodologías propuestas por el CAB en los documentos: "Cuenta Satélite de Cultura: manual metodológico para su implementación en Latinoamérica" (2009) y "Guía de Implementación Metodológica para las Cuentas Satélite de Cultura en Iberoamérica" (2015).

El Manual del CAB en su versión 2009, ha sido el marco más utilizado por los países. En este grupo se encuentran: Bolivia, Colombia, Costa Rica, Ecuador, Guatemala, México, Perú y Uruguay. De otra parte, el MEC es usado como base conceptual en las mediciones de Chile, España, México, República Dominicana y Uruguay. Además, la Guía Metodológica del CAB en su versión 2015, ha sido implementada sólo por Colombia y República Dominicana.

Es importante resaltar, que los países de la Región ubicados en Europa han implementado marcos distintos. Por ejemplo, España utiliza en sus bases metodológicas el Sistema Europeo de Cuentas, el informe final sobre estadísticas culturales del Leadership Group on Culture Statistics (LEG) y el informe final en Cultura del European Statistical System Network on Culture (ESSnet), estos 
últimos difundidos por la Comisión Europea en 2010 y 2012, respectivamente. Por otro lado, Portugal utiliza para la medición de las cuentas el Manual del Sistema Europeo de Cuentas Nacionales y Regionales SEC 2010 y el Sistema Estadístico Europeo para la Red Cultural de 2012. Es el caso también de Chile, que acoge los lineamientos de la OCDE para la implementación de las CSC, y México que tiene en cuenta las recomendaciones de la Organización Mundial de la Propiedad Intelectual (OMPI).

Existen otros países en la región que utilizan adicionalmente los marcos de sus cuentas nacionales o el Manual del SCN 2008; es el caso de Argentina, Colombia, Costa Rica, México y República Dominicana.

De otra parte, las clasificaciones y nomenclaturas más utilizadas son la CIIU y la CPC para actividades y productos culturales, respectivamente, tal como lo recomiendan el MEC y los documentos del CAB; sin embargo muchos de los países no han adaptado sus mediciones a las revisiones más actuales. Por ejemplo: Chile y Colombia, utilizan la CIIU Rev 3; Argentina, Bolivia, Colombia, Ecuador, Guatemala y Perú utilizan revisiones anteriores a la CPC Rev 2. España, Portugal y México por su lado, utilizan clasificaciones distintas a las del resto de países de la región, en ese sentido su comparabilidad con los países de la región es inviable sin la realización de un trabajo estadístico adicional.

Con relación al alcance temático, los datos confirman que la mayoría de países de la región ha avanzado en la medición de la producción y el valor agregado cultural; excepto República Dominicana, que inició su proceso de elaboración con el análisis del consumo cultural y del gasto, todos los países cuentan con resultados sobre producción y valor agregado, tal como lo muestra la siguiente nube de frecuencias:

\section{Nube de palabras más frecuentes en la variable alcance temático}

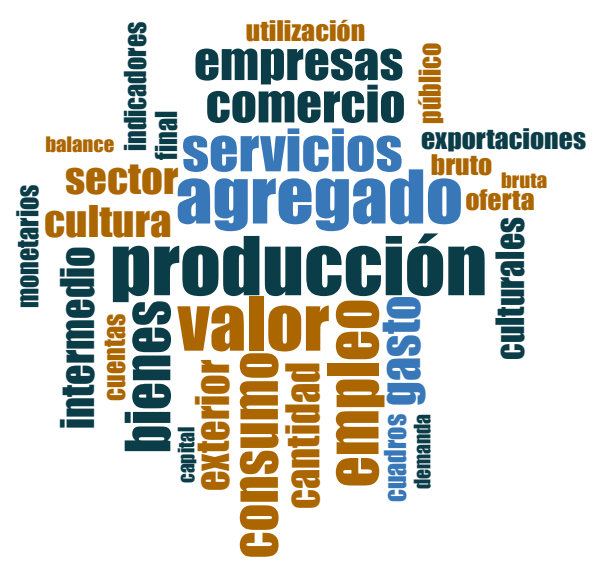

Fuente: elaboración propia, NVIVO 
Esto puede estar explicado por varias razones, una de ellas es que varios países de la región han iniciado recientemente sus procesos de medición; en ese sentido la disponibilidad de información sobre la oferta es mayor debido a que una proporción importante de países cuentan con estadísticas económicas que facilitan el cálculo de indicadores por esta vía. Otra de las razones es estratégica, los países que han enfocado su medición en la producción y, en consecuencia en el valor agregado, han ganado visibilidad frente a otros sectores, por el cálculo de su contribución a la economía en su conjunto. Lo mismo sucede con la variable de empleo, el cálculo del aporte a la fuerza productiva de un país es un dato relevante cuando se requiere la negociación de la cartera del sector.

Las variables que les siguen en importancia a la producción y al valor agregado son empleo y comercio exterior, ya que el 69 y $62 \%$ de los países respectivamente, han realizado mediciones sobre este tipo de información. Adicionalmente se registra un porcentaje importante para el análisis del gasto, pues el 54\% de los países lo han abordado en la medición de las CSC.

Respecto a las limitaciones que presentan los países para la elaboración de las cuentas se encontró con mayor frecuencia la escasa disponibilidad de información para el sector cultural, la desagregación inadecuada para obtener datos de los subsectores y la informalidad característica de la actividad cultural, lo que dificulta la obtención de información.

Por último, los países plantean como principal desafío el uso de los datos generados para la toma de decisiones en el diseño y análisis de las políticas públicas y en la distribución eficiente de los recursos.

\subsubsection{Matriz de avances en cálculos y cobertura}

La segunda matriz comprende las variables de año de la base económica, serie disponible, año de publicación más reciente, contribución del valor agregado a la economía nacional y sectores cubiertos.

El análisis de los datos muestra que los años de las bases económicas que se han utilizado como referencia para los cálculos de las CSC, están entre el año 2001 y el año 2011. Guatemala y Argentina utilizan bases 2001 y 2004, respectivamente. Los países con bases más recientes son Costa Rica y Portugal. El año que aparece con mayor frecuencia como base económica es el 2007, esto se debe a que un grupo de países elaboraron de forma conjunta las CSC, ellos son: Ecuador, Perú y Bolivia. Por otro lado, la serie de datos disponibles más largas las tienen Argentina, Colombia y España, las cuales abarcan 10 años o más de medición. 
El año de publicación de resultados más reciente es 2016 correspondiente a 9 de los 13 países que conforman este bloque. El año más alejado de publicación lo reporta Chile, ya que fue en 2010 cuando divulgó un documento con los datos que se aproximan a las Cuentas Satélite de Cultura.

Las contribuciones del valor agregado cultural a las economías nacionales van desde 0,6\% en Uruguay hasta el 3,1\%, en España. La mediana de las contribuciones a las economías se sitúa en el 1,6\% y la media en el 1,8\%, lo que muestra una distribución asimétrica en los datos del valor agregado por el momento, que se puede explicar por las diferentes coberturas de medición alcanzada en los países.

En ese sentido, se evidencia que ninguno de los países ha cubierto el $100 \%$ de los sectores propuestos por la Guía del CAB (2015) o el MEC de UNESCO (2009). Aun así se destacan Colombia con el $82 \%$ de la cobertura alcanzada, España con el 73\%; y, Argentina, Costa Rica, Portugal y República Dominicana, quienes han cubierto más del 50\% del campo cultural en sus mediciones. Es importante resaltar que la medición de un sector aunque haya sido cubierto puede ser parcial por la disponibilidad de la información, por la desagregación en el país, lo que a su vez puede producir subestimación en los sectores.

En este grupo de países los sectores medidos con mayor frecuencia son Libros y Publicaciones, Artes Escénicas y Audiovisual y Radio, estos sectores son preferidos por la disponibilidad de información y por ser nucleares dentro del campo cultural. Los sectores que plantean un desafío de medición para los países son patrimonio inmaterial y creación, esto ocurre debido a la dificultad metodológica para su medición que aún se encuentra en discusión y la disponibilidad de información para acercarse a su valoración.

\subsubsection{Matriz de fuentes de información}

La tercera matriz contiene datos sobre los tipos de operaciones estadísticas utilizadas en las CSC, las entidades encargadas de generarlas y los años disponibles. De esta manera, se comprobó que las fuentes más utilizadas corresponden a las encuestas y los registros administrativos. Lo que sugiere para los países de la región el fortalecimiento de la generación de datos con operaciones menos costosas, y el aprovechamiento de los datos que se generan por diferentes conceptos en las administraciones públicas.

\section{Nube de frecuencias de las fuentes de información de las CSC}




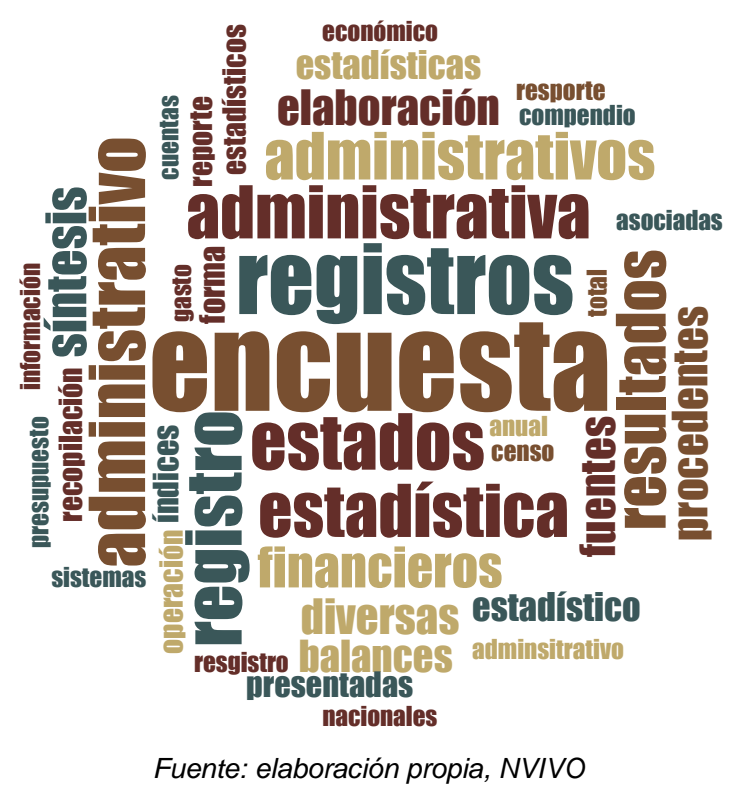

Se encontró además que las entidades productoras de este tipo de información son los institutos de estadísticas oficiales en la mayoría de los casos; así las cosas, una alianza clave para los responsables de elaborar las CSC se prevé con estas instituciones.

En relación con los períodos disponibles, se evidencia en los años recientes una producción continuada de los datos, en algunos casos las periodicidades de producción son anuales, lo cual garantizaría resultados permanentes, sin embargo en otros casos las periodicidades son bianuales o cada tres años. En ese sentido se sugiere establecer una periodicidad de las cuentas de acuerdo con la existente en la producción estadística de base para su elaboración.

\subsection{Países en proceso de implementación}

De otra parte, el grupo que se encuentra en proceso de implementación o interesados en la elaboración de las cuentas satélite de cultura está conformado por: Brasil, El Salvador, Honduras, Panamá y Paraguay. Este grupo de países se encuentra actualmente en los trabajos preparatorios para la elaboración de las CSC.

En Brasil, la institucionalidad encargada de las cuentas está conformada por el Ministerio de Cultura, el Instituto Brasilero de Geografía y Estadística y la Universidad de San Pablo. Como trabajo preparatorio para la elaboración de las cuentas se destacan dos documentos técnicos. El primero contiene la cartografía y el análisis de operaciones estadísticas, que generan información relevante para las cuentas satélite de cultura; el segundo contiene un mapeo detallado de la información complementaria e indicadores requeridos para la elaboración de las CSC. Adicionalmente el país cuenta con un cuaderno metodológico, bases de datos culturales y los 
cuadernos de consolidación histórica, los cuales tienen métodos y datos útiles para la elaboración de las cuentas.

En El Salvador la Dirección General de Estadística y Censo es la entidad a cargo de la elaboración de las CSC, se destacan los esfuerzos realizados para la generación de información básica mediante un sistema que "comprende las actividades culturales y tienen como principal finalidad, reunir en un cuadro estadístico el conjunto de cifras relacionadas con la actividad del campo cultural objeto de investigación, como son bibliotecas, museos, sitios arqueológicos, radiodifusoras, entre otros" (DIGESTYC, consultado el 3 de marzo de 2017). En la actualidad están disponibles los datos de 2005-2010 de bibliotecas universitarias, nacionales, lectores por sexo y obras consultadas. De otra parte también se encuentra información sobre visitas a bibliotecas, préstamos y obras consultadas.

Los avances de Honduras en la medición de la cultura se realizan a través de la Secretaría de la Cultura, Artes y Deportes y el Banco Central de Honduras. Se ha logrado consolidar un Sistema de Información Cultural en el país, que recolecta información sobre los agentes y las instituciones culturales que pueden servir como insumo para la construcción de directorios y el levantamiento de información básica para las CSC.

En Panamá durante 2015, el Instituto Nacional de Estadística y Censo (INEC), realizó el marco de estadísticas culturales del país, mediante una investigación sobre las operaciones y registros administrativos disponibles del sector cultural, que identificó la información básica necesaria para la elaboración de las cuentas. El trabajo realizado permitió además proponer un marco metodológico de base para la medición de la cultura. Las conclusiones del documento señalan que si bien existe información disponible que puede apoyar el cálculo de las CSC, se debe generar información adicional en el país. Durante 2016, el Equipo de Estadísticas Sociales de INEC inició los trabajos de recolección de información de datos de cultura que serán los insumos para las cuentas y en 2017 se espera la primera publicación de resultados de la información cultural básica y el inicio de los trabajos de las CSC en Panamá.

La Secretaría Nacional de Cultura (SNC) es la institución responsable de las políticas públicas culturales en Paraguay. En diciembre de 2014, se llevó a cabo una jornada sobre las Cuentas Satélite de Cultura organizada por la SNC con el apoyo de la Comisión Económica para América Latina y el Caribe (CEPAL), bajo el propósito de convocar a los actores involucrados en la generación de información requerida para la elaboración de las CSC, dentro de los cuales se identificó el Sistema Nacional de Cultura del Paraguay, la Dirección General de Encuestas, Estadísticas y Censos, el Ministerio de Educación, Secretaría de Estado de Tributación, entre otros (SCN, consultado el 2 de marzo de 2017). 
En dicho encuentro se presentó la información cultural disponible para este trabajo, y se propuso la instalación de una mesa técnica de carácter interinstitucional e intersectorial de intercambio de conocimiento e información con el fin de iniciar el proceso de elaboración de las cuentas (SCN, consultado el 2 de marzo de 2017). Asimismo en 2015 se realizó un "Seminario Regional de Cuenta Satélite de Cultura" que contó con el apoyo de CEPAL, OEI y la participación de expertos de América Latina. Los inicios de los trabajos se formalizaron durante diciembre de 2015 liderada por la SNC y apoyada por las instituciones involucradas con el sector cultural, y las que recolectan información o producen registros administrativos que sirven de insumo para la elaboración de las CSC. Se decidió además, que el proceso de medición de estas cuentas inicie con la medición del Sector Audiovisual, trabajo que se encuentra aún en desarrollo junto a la medición de otros sectores culturales (SCN, consultado el 2 de marzo de 2017).

En resumen, el grupo de países que han iniciado el proceso de implementación ya cuentan con avances en la medición de las CSC pues han conformado equipos de trabajo, realizado actividades de mapeo e identificación de datos disponibles o posibles de ser generados en el corto plazo, y explorado conceptualmente el campo cultural.

Dentro de la región hay tres países sobre los que no pudo establecer si están interesados o se han realizado avances sobre la medición de las CSC, estos son: Cuba, Nicaragua y Venezuela. No obstante, Cuba ha generado un sistema de indicadores culturales en el Ministerio de Cultura adecuado para la economía del país (en su gran mayoría de no mercado) y Venezuela cuenta con operaciones estadísticas culturales de demanda cultural que pueden ser un insumo importante para su desarrollo.

\section{Conclusiones y acciones recomendadas para el desarrollo y sostenibilidad de las CSC}

Los resultados obtenidos permiten confirmar tres desafíos importantes que enfrentan los responsables de las CSC para su desarrollo y sostenibilidad:

En primer lugar se identificó que los países de lberoamérica que han implementado las CSC no han logrado la cobertura total de la medición debido a las características de la información de base, no sólo por su insuficiencia o inexistencia sino también por su desagregación, oportunidad y disponibilidad. En ese sentido se propone como una acción de política pública para mejorar la información del sector, realizar de manera conjunta a la construcción de las cuentas, procesos de planificación estadística del sector. El proceso de planificación estadística permite eliminar las asimetrías de información entre productores de datos básicos, usuarios de la información y tomadores de decisión; actualizar de manera permanente los sistemas estadísticos; fortalecer las 
estadísticas básicas; evitar duplicidad de esfuerzos en la producción de datos básicos; y, aumentar la confiabilidad del sector cultural y de las estadísticas nacionales en general.

El segundo desafío corresponde a la viabilidad de las comparaciones internacionales, que se evidencian por la utilización de diferentes bases conceptuales y metodológicas para la elaboración de las cuentas satélite de cultura. En ese sentido se plantea como acción estratégica la unificación conceptual y metodológica mediante un marco de medición conceptual y metodológico, de carácter global, que admita un núcleo básico de comparabilidad y al mismo tiempo, mediciones alternativas que se adapten a las necesidades y particularidades de cada país sobre el sector cultural. De esta manera no solo se alcanzaría la comparabilidad entre naciones, sino el desarrollo de iniciativas para unir esfuerzos de desarrollo del sector cultural entre países con características similares.

Por último y no menos importante, es lo referido al uso de la información generada en las CSC para el objeto por el cual se ha apoyado su construcción. El bloque iberoamericano ha constatado la importancia de contar con este tipo de información, pero aún se observan muy pocos ejercicios de utilización de esta información en avances investigativos para el desarrollo del sector o para la toma de decisiones en las políticas públicas y privadas. Por eso como una de las acciones consiste en desarrollar una estrategia de divulgación y difusión efectiva e inteligente de la información que se produce en las cuentas satélite de cultura y además proponer las formas de articulación de los datos y su utilidad para el diseño, monitoreo y evaluación de las políticas públicas culturales.

Las tres recomendaciones que se plantean tienen alta probabilidad de implementación porque implican además hacer uso eficiente de los recursos disponibles, aumentar la credibilidad de la cultura y mejorar la calidad de la atención de las intervenciones públicas y privadas en el sector cultural.

En conclusión, es posible afirmar que no obstante las cuentas satélite de cultura requieren de algunos ajustes, constituyen un sistema de información muy completo que permite conocer la situación del sector cultural en los países y de acuerdo con los resultados de la investigación, Iberoamérica se constituye en la región con más experiencia en este tipo de mediciones, que puede considerarse como una propuesta y apuesta para los avances en la producción de información cultural en el mundo. 


\section{Bibliografía}

ARGENTINA (2016). Cuenta Satélite de Cultura Metodología: Buenos Aires: Sistema de Información Cultural de La Argentina \& Ministerio de Cultura. Recuperado de: http://www.sinca.gob.ar/sic/estadisticas/csc/index.php

AUSTRALIAN BUREAU OF STATISTICS (2014). Australian National Accounts: Cultural and Creative Activity Satellite Accounts. Recuperado de:

http://www.abs.gov.au/ausstats/abs@.nst/0/C0F8EF251D42B881CA257C78000C1AA7?Op endocument

BORDIEU (1966). Campo Intelectual, Campo de Poder. Buenos Aires: Editorial Montressor

CEPAL \& OEI (2015). Cultura y Desarrollo Económico en Iberoamérica. Santiago de Chile: CEPAL $\& O E I$

CHILE (2012). Documento metodológico: una aproximación económica a la cultura en Chile. Santiago de Chile: Consejo Nacional de la Cultura y las Artes. Recuperado de: http://www.cultura.gob.cl/wp-content/uploads/2012/03/metodologia-aproximacioneconomica.pdf

COLOMBIA (2016). Cuenta Satélite de Cultura de Colombia. Bogotá: DANE y Ministerio de Cultura. Recuperado de:

http://www.dane.gov.co/index.php/estadisticas-por-tema/cuentas-nacionales/cuentassatelite/cuenta-satelite-de-cultura-en-colombia/cuenta-satelite-de-cultura-2005-2014

CONVENIO ANDRÉS BELLO (2009). Cuenta Satélite de Cultura: Manual Metodológico para su Implementación en Latinoamérica. Bogotá: D’Vini

CONVENIO ANDRÉS BELLO (2015). Guía Metodológica para la Implementación de las Cuentas Satélite de Cultura en Iberoamérica. Bogotá: Editorial Panamericana

COSTA RICA (2016). Cuenta Satélite de Cultura de Costa Rica. San José: Ministerio de Cultura y Juventud. Recuperado de: https://cuentasatelitecultura.go.cr/resultados/

ESPAÑA (2016). Cuenta Satélite de Cultura de España. Madrid: Ministerio de Educación, Cultura y Deporte. Recuperado de: 
http://www.mecd.gob.es/servicios-al-ciudadano-

mecd/estadisticas/cultura/mc/csce/portada.html

GUATEMALA (2016). La Cuenta Satélite de Cultura en Guatemala. Guatemala: Ministerio de Cultura y Deportes de la República de Guatemala. Recuperado de: http://mcd.gob.gt/lacuenta-satelite-de-cultura-de-guatemala-esta-en-marcha/

MEXICO (2015). Cuenta Satélite de Cultura de México. México D.F.: INEGI \& CONACULTA. Recuperado de:

http://internet.contenidos.inegi.org.mx/contenidos/productos//prod serv/contenidos/espanol/ bvinegi/productos/derivada/satelite/cultura/702825056575.pdf

NACIONES UNIDAS, et al. (2008). Sistema de Cuentas Nacionales 2008. Santiago de Chile: CEPAL.

NATIONAL ENDOWMENT FOR THE ARTS (2013). Guide to the U.S. Arts and Cultural. Washington, D.C.: NEA. Recuperado de:

https://www.arts.gov/sites/default/files/nea_guide_white_paper.pdf

PORTUGAL (2016). Conta Satélite da Cultura 2010-2012. Lisboa: Instituto Nacional de Estatística. Recuperado de: http://www.gepac.gov.pt/estatisticas-e-estudos/estatisticas.aspx

POTTS, J. \& CUNNINGHAM, S. (2008). Four models of the creative industries. International Journal of Cultural Policy, 14(3): 233-49.

REPÚBLICA DOMINICANA (2016). Cuenta Satélite de Cultura de la República Dominicana. Santo Domingo: Ministerio de Cultura y Banco Central. Recuperado de:

http://www.bancentral.gov.do/noticias/otras_noticias/archivos/bc2016-05-

05_Informe_CSC_RD.pdf

STATISTICS FINLAND (2016). Culture Satellite Account. Recuperado de:

http://www.stat.fi/til/klts/index en.html

UNESCO (2009). Marco de Estadísticas Culturales 2009. Montreal: UIS UNESCO

URUGUAY (2015). Cuenta Satélite en Cultura del Uruguay. Montevideo: Dirección Nacional de Cultura. Recuperado de: 


\section{Breve perfil profesional de la autora}

Ingeniera industrial de la Universidad del Norte, con maestría en políticas públicas de la Universidad de los Andes y posgrado en gerencia y gestión cultural de la Universidad del Rosario. Trabajó durante 9 años en el DANE como responsable de las cuentas satélite de cultura de Colombia. Es coautora de la 'Guía Metodológica para la implementación de las Cuentas Satélite de Cultura en Iberoamérica' publicada por el Convenio Andrés Bello y de la 'Metodología de la cuentas satélite de cultura de Colombia'. Además es autora de varios artículos sobre los sistemas de información del campo cultural. Fue miembro del grupo de trabajo que discutió y elaboró la propuesta del Marco de Estadísticas Culturales (MEC) de UNESCO. Ha participado en la transferencia del conocimiento para la elaboración de las cuentas satélite de cultura de Cuba, Brasil, Chile, Perú, Argentina, Uruguay, Honduras, España, Costa Rica y Ecuador; y en el diseño de los sistemas de información cultural de Ecuador, República Dominicana y Panamá. Actualmente se desempeña como funcionaria de la Auditoría General de la República, entidad en la que diseña y coordina la implementación de políticas públicas de control fiscal, elabora metodologías, recomendaciones y políticas de gestión y vigilancia fiscal de los recursos destinados a sectores económicos, tales como la cultura; y está vinculada como docente catedrático del pregrado y posgrado de la Facultad de Estudios de Patrimonio Cultural en la Universidad Externado de Colombia. 